\title{
Efficient quantum protocols for XOR functions
}

\author{
Shengyu Zhang
}

\begin{abstract}
We show that for any Boolean function $f:\{0,1\}^{n} \rightarrow\{0,1\}$, the bounded-error quantum communication complexity $\mathrm{Q}_{\epsilon}(f \circ \oplus)$ of XOR functions $f(x \oplus y)$ satisfies that $\mathrm{Q}_{\epsilon}(f \circ \oplus)=$ $O\left(2^{d}\left(\log \|\hat{f}\|_{1, \epsilon}+\log \frac{n}{\epsilon}\right) \log (1 / \epsilon)\right)$, where $d=\operatorname{deg}_{2}(f)$ is the $\mathbb{F}_{2}$-degree of $f$, and $\|\hat{f}\|_{1, \epsilon}=$ $\min _{g:\|f-g\|_{\infty} \leq \epsilon}\|\hat{g}\|_{1}$. This implies that the previous lower bound $Q_{\epsilon}(f \circ \oplus)=\Omega\left(\log \|\hat{f}\|_{1, \epsilon}\right)$ by Lee and Shraibman [LS09] is tight for $f$ with low $\mathbb{F}_{2}$-degree. The result also confirms the quantum version of the Log-rank Conjecture for low-degree XOR functions. In addition, we show that the exact quantum communication complexity satisfies $Q_{E}(f)=O\left(2^{d} \log \|\hat{f}\|_{0}\right)$, where $\|\hat{f}\|_{0}$ is the number of nonzero Fourier coefficients of $f$. This matches the previous lower bound $Q_{E}(f(x, y))=\Omega\left(\log \operatorname{rank}\left(M_{f}\right)\right)$ by Buhrman and de Wolf BdW01] for low-degree XOR functions.
\end{abstract}

\section{Introduction}

Communication complexity studies the minimum amount of communication needed for a computational task with input distributed to two (or more) parties. Communication complexity has been applied to prove impossibility results for problems in a surprisingly wide range of computational models. At the heart of studies of communication complexity are lower bounds, and the tightness of lower bound techniques has been among the most important, and at the same time, most challenging questions. Indeed, one of the most famous open problems in communication complexity is the Log-rank Conjecture: It has been known that the (two-party, interactive) deterministic communication complexity $\mathrm{D}(f) \geq \log _{2}\left(\operatorname{rank}\left(M_{f}\right)\right)$ [MS82], where the rank is over $\mathbb{R}$ and $M_{f}$ is the communication matrix defined as $M_{f}(x, y)=f(x, y)$. The Log-rank Conjecture, proposed by Lovász and Saks [LS88], says that the above bound is polynomially tight, namely

$$
\mathrm{D}(f)=O\left(\log _{2}^{O(1)}\left(\operatorname{rank}\left(M_{f}\right)\right)\right) .
$$

A quantum version of the conjecture, also seemingly hard to attack, says that the $\epsilon$-bounded error quantum communication complexity

$$
\mathrm{Q}_{\epsilon}(f)=O\left(\log _{2}^{O(1)}\left(\operatorname{rank}_{\epsilon}\left(M_{f}\right)\right)\right),
$$

where $\operatorname{rank}_{\epsilon}\left(M_{f}\right)=\min \left\{\operatorname{rank}_{\epsilon}\left(M_{f}\right):\|f-g\|_{\infty} \leq \epsilon\right\}$ [BdW01, LS09]. Note that proving this type of conjectures needs to design efficient communication protocols.

Communication complexity for the class of XOR functions has recently drawn an increasing amount of attention [ZS09, ZS10, LZ10, MO10, LLZ11, SW12, LZ13, TWXZ13]. The class contains those functions $F(x, y)=f(x \oplus y)$ for some function $f:\{0,1\}^{n} \rightarrow\{0,1\}$, where the inner operator $\oplus$ is the bit-wise XOR. Denote such functions $F$ by $f \circ \oplus$. This class includes important functions 
such as Equality (deciding whether $x=y$ ) Yao79, NS96, Amb96, BK97, BCWdW01]), Hamming Distance (deciding whether $|x \oplus y| \leq d$ ) [Yao03, GKdW04, HSZZ06, ZS09, LLZ11, LZ13, and Gap Hamming Distance (distinguishing $|x \oplus y| \leq n / 2-\sqrt{n}$ and $|x \oplus y| \geq n / 2+\sqrt{n}$ ) [JKS08, CR12, She12, Vid12. Communication complexity of XOR functions also exhibits interesting connections to Fourier analysis of Boolean functions. First, the rank of the communication matrix $M_{f}$ is nothing but $\|\hat{f}\|_{0}$, the number of nonzero Fourier coefficients of $f$. Thus for XOR functions, the Log-rank Conjecture becomes the assertion that $\mathrm{D}(f \circ \oplus)=O\left(\log O(1)\|\hat{f}\|_{0}\right)$; see [ZS09, MO10, KS13, TWXZ13, for some investigations on this topic. The quantum Log-rank Conjecture becomes $\mathrm{Q}_{\epsilon}(f)=O\left(\log _{2}^{O(1)}\left(\|\hat{f}\|_{0, \epsilon}\right)\right)$ accordingly, where $\|\hat{f}\|_{0, \epsilon}=\min \left\{\|\hat{g}\|_{0}:\|f-g\|_{\infty} \leq \epsilon\right\}$. Second, as shown in [LS09], the quantum communication complexity for computing $f(x \oplus y)$ is known to be lower bounded by an approximate version of the Fourier $\ell_{1}$-norm as follows.

$$
\mathrm{Q}_{\epsilon}(f \circ \oplus)=\Omega\left(\log \|\hat{f}\|_{1, \epsilon}\right), \text { where }\|\hat{f}\|_{1, \epsilon}=\min \left\{\|\hat{g}\|_{1}:\|f-g\|_{\infty} \leq \epsilon\right\} .
$$

The tightness of this lower bound has been an intriguing question.

In this paper, we show that the bound in Eq.(3) is tight for functions $f$ with low $\mathbb{F}_{2}$-degree, the degree of $f$ viewed as a polynomial in $\mathbb{F}_{2}\left[x_{1}, \ldots, x_{n}\right]$. For convenience of comparison, we copy the lower bound in Eq.(3) into the following theorem.

Theorem 1. For any function $f:\{0,1\}^{n} \rightarrow\{0,1\}$ with $\operatorname{deg}_{2}(f)=d$, and any $\epsilon \in\left(0,1 / 2^{d+4}\right)$, we have

$$
\Omega\left(\log \|\hat{f}\|_{1, \epsilon}\right) \leq \mathrm{Q}_{\epsilon}(f \circ \oplus) \leq O\left(2^{d}\left(\log \|\hat{f}\|_{1, \epsilon}+\log \frac{n}{\epsilon}\right) \log (1 / \epsilon)\right) .
$$

This theorem has two implications on the Log-rank Conjectures. First, it was known that the above lower bound was smaller than $\log \operatorname{rank}_{\epsilon}\left(M_{f \circ \oplus}\right)$, and the above upper bound satisfies $\|\hat{f}\|_{1, \epsilon} \leq\|\hat{f}\|_{0, \epsilon}$. Thus the above upper bound confirms the quantum Log-rank Conjecture (Eq.(2) ) for low-degree XOR functions.

Corollary 2. The quantum Log-rank Conjecture holds for XOR functions $f$ with $\mathbb{F}_{2}$-degree at most $O\left(\log \log \|\hat{f}\|_{1, \epsilon}\right)$.

Second, we also have a variant of the protocol in Theorem 1, and the variant is an exact protocol in the sense that it has a fixed number of qubits exchanged besides that it has zero error. (For comparison, the classical zero-error protocols usually refer to Las Vegas ones in which the number of bits exchanged is a random variable that can be very large, and the complexity cost measure is the expectation of the number of communication bits.) For exact protocols, we have the following theorem, where the lower bound is from [BdW01]; we copy it into the following theorem, again for the convenience of comparison.

Theorem 3. For any function $f:\{0,1\}^{n} \rightarrow\{0,1\}$ with $\operatorname{deg}_{2}(f)=d$, we have

$$
\frac{1}{2} \log _{2}\|\hat{f}\|_{0} \leq \mathrm{Q}_{E}(f \circ \oplus) \leq 2^{d+1} \log _{2}\|\hat{f}\|_{0} .
$$

In particular, $\mathrm{Q}_{E}(f \circ \oplus)$ is polynomially related to $\log \operatorname{rank}\left(M_{f \circ \oplus}\right)$ when $\operatorname{deg}_{2}(f)=O\left(\log \log \|\hat{f}\|_{0}\right)$.

In [TWXZ13, it shows that $\mathrm{D}(f \circ \oplus) \leq O\left(2^{d^{2} / 2} \log ^{d-2}\|\hat{f}\|_{0}\right)$, which implies that the Log-rank Conjecture holds for constant-degree XOR functions. The complexity bound in Theorem 3 has a 
better dependence on $d$, which enables us to obtain an upper bound of $\log O(1)\|\hat{f}\|_{0}$ for a larger range of $d$. Another desirable property of the protocol in Theorem 3 is that unlike the ones in TWXZ13] and most other upper bounds in communication complexity, this protocol is efficient not only in communication but also in computation, provided that the Fourier spectrum can be efficiently encoded and decoded.

Techniques One common idea the protocols in this paper share with the ones in [TWXZ13] (as well as many work in additive combinatorics) is degree reduction: The protocols have $d$ rounds and each round $i$ reduces the problem of computation of a function $f_{i}$ to that of another function $f_{i+1}$, with $\operatorname{deg}_{2}\left(f_{i+1}\right) \leq \operatorname{deg}_{2}\left(f_{i}\right)-1$. Different than the protocols in [TWXZ13, the protocol in this paper are not derived from parity decision tree algorithms. Neither do they use linear polynomial rank or analyze any effect of linear restrictions on the Fourier domain as in [TWXZ13. Instead, the protocols in this paper merely use the definition of quantum Fourier transform over the additive group of $\mathbb{F}_{2}^{n}$, and the efficiency of the protocols comes directly from the Fourier sparsity of the corresponding function. Some new difficulty appears in this quantum Fourier sampling approach: $f_{i+1}$ is actually known only to Alice but not to Bob. This is solved by observing a simple (yet important) property of the collection of derivatives of $f_{i}$ along all directions.

\section{Preliminaries}

Let $[n]=\{1,2, \ldots, n\}$. For a vector $v \in \mathbb{R}^{N}$, its $\operatorname{support}$ is $\operatorname{supp}(v)=\left\{i \in[N]: v_{i} \neq 0\right\}$. For two $n$-bit strings $x$ and $y$, their addition, denoted $x \oplus y$ (or sometimes just $x+y$ ), is bit-wise over $\mathbb{F}_{2}$. For a set $A \subseteq\{0,1\}^{n}$, define $A+A=\left\{a_{1}+a_{2}: a_{1}, a_{2} \in A\right\}$. In general, define $k A=\left\{a_{1}+\cdots+a_{k}: a_{i} \in A, \forall i \in[k]\right\}$. It is easy to see that $|k A| \leq|A|^{k}$.

A Boolean function $f:\{0,1\}^{n} \rightarrow\{0,1\}$ can be viewed as a multi-linear polynomial over $\mathbb{F}_{2}$, whose degree is called $\mathbb{F}_{2}$-degree and denoted by $\operatorname{deg}_{2}(f)$. For a function $f:\{0,1\}^{n} \rightarrow\{0,1\}$ and a direction vector $t \in\{0,1\}^{n}-\left\{0^{n}\right\}$, the derivative $\Delta_{t} f$ is defined by $\Delta_{t} f(x)=f(x)+f(x+t)$, where both additions are over $\mathbb{F}_{2}$. It is easy to check that $\operatorname{deg}_{2}\left(\Delta_{t} f\right)<\operatorname{deg}_{2}(f)$ for any non-constant $f$ and any $t \in\{0,1\}^{n}-\left\{0^{n}\right\}$. If one represents the range of a Boolean function by $\{+1,-1\}$, the derivative becomes $\Delta_{t} f(x)=f(x) f(x+t)$.

For a real function $f:\{0,1\}^{n} \rightarrow \mathbb{R}$, one can define its Fourier coefficients by $\hat{f}(\alpha)=2^{-n} \sum_{x} f(x) \chi_{\alpha}(x)$, where the characters $\chi_{\alpha}(x)=(-1)^{\alpha \cdot x}$ are orthogonal with respect to the inner product $\left\langle f_{1}, f_{2}\right\rangle=$ $2^{-n} \sum_{x}\left[f_{1}(x) f_{2}(x)\right]$. The function $f$ can be written as $f=\sum_{\alpha} \hat{f}(\alpha) \chi_{\alpha}$. The Fourier sparsity of $f$, denoted by $\|\hat{f}\|_{0}$, is the number of nonzero Fourier coefficients of $f$. For any $p>0$, the $\ell_{p^{-}}$ norm of $\hat{f}$, denoted by $\|\hat{f}\|_{p}$, is $\left(\sum_{\alpha}|\hat{f}(\alpha)|^{p}\right)^{1 / p}$. In particular, $\|\hat{f}\|_{1}=\sum_{\alpha}|\hat{f}(\alpha)|$. One can also define an approximate version of the Fourier $\ell_{1}$-norm by $\|\hat{f}\|_{1, \epsilon}=\min \left\{\|\hat{g}\|_{1}:\|f-g\|_{\infty} \leq \epsilon\right\}$ where $\|f-g\|_{\infty}=\max _{x}|f(x)-g(x)|$. Similarly define $\|\hat{f}\|_{0, \epsilon}=\min \left\{\|\hat{g}\|_{0}:\|f-g\|_{\infty} \leq \epsilon\right\}$.

For any function $f:\{0,1\}^{n} \rightarrow \mathbb{R}$, Parseval's Indentity says that $\sum_{\alpha} \hat{f}_{\alpha}^{2}=\mathbf{E}_{x}\left[f(x)^{2}\right]$. When the range of $f$ is $\{+1,-1\}$, this becomes $\sum_{\alpha} \hat{f}_{\alpha}^{2}=\mathbf{E}_{x}\left[f(x)^{2}\right]=1$.

The quantum Fourier transform on $\mathbb{F}_{2}^{n}$ is defined by $\sum_{x} c_{x}|x\rangle \mapsto 2^{-n / 2} \sum_{x, \alpha} c_{x} \chi_{\alpha}(x)|\alpha\rangle$, and it is easily seen to be a unitary operator. The transform can be implemented by $H^{\otimes n}$ where $H=\frac{1}{\sqrt{2}}\left(\begin{array}{cc}1 & 1 \\ 1 & -1\end{array}\right)$ is the Hadamard matrix.

The following lemma extends Chernoff's bound to general domains; see, for example, DP12] (Problem 1.19). 
Lemma 4. Suppose we have random variables $X_{i} \in\left[a_{i}, b_{i}\right]$ for $i=1,2, \ldots, n$, and let $X=\sum_{i=1}^{n} X_{i}$. Then

$$
\operatorname{Pr}[|X-\mathbf{E}[X]|>t]<2 e^{-\frac{2 t^{2}}{\sum_{i}\left(b_{i}-a_{i}\right)^{2}}} .
$$

In particular, if each $X_{i}$ takes values in $[-1,1]$, then

$$
\operatorname{Pr}[|X-\mathbf{E}[X]|>t]<2 e^{-\frac{t^{2}}{4 n}} .
$$

\section{Protocol}

In this section, we will show Theorem 1. We will first mention how to convert Fourier $\ell_{1}$-norm to Fourier $\ell_{0}$-norm in Section 3.1, and then show the main protocol in Section 3.2.

\subsection{From $\ell_{1}$-norm approximation to $\ell_{0}$-norm approximation}

In [BS91, Gro97], the sampling of characters with probability proportional to Fourier coefficients (in abstract value) is studied. Given a function $f:\{0,1\}^{n} \rightarrow \mathbb{R}$, we sample $\alpha \in\{0,1\}^{n}$ with probability $|\hat{f}(\alpha)| /\|\hat{f}\|_{1}$. We refer to a sample from this process as a Fourier $\ell_{1}$-sample. Using Lemma 4, it is not hard to show the following lemma.

Lemma 5 (Grolmusz, Gro97]). For a function $g:\{0,1\}^{n} \rightarrow \mathbb{R}$, independently draw $M=$ $O\left(\|\hat{g}\|_{1}^{2} n \log (1 / \lambda) / \delta^{2}\right)$ Fourier $\ell_{1}$-samples $\alpha^{1}$, .., $\alpha^{M}$. Let $h(x)=\frac{\|\hat{g}\|_{1}}{M} \sum_{i=1}^{M} \operatorname{sign}\left(\hat{g}\left(\alpha^{i}\right)\right) \chi_{\alpha^{i}}(x)$. Then

$$
\operatorname{Pr}\left[\forall x \in\{0,1\}^{n},|h(x)-g(x)| \leq \delta\right] \geq 1-\lambda .
$$

The original lemma actually considers the probability of $\operatorname{sign}(h(x))=\operatorname{sign}(g(x))$, but the same proof works for the above statement. We include a proof here for completeness.

Proof. Let $Z_{i}=\operatorname{sign}\left(\hat{g}\left(\alpha^{i}\right)\right) \chi_{\alpha^{i}}(x) \in\{+1,-1\}$. Note that

$$
\mathbf{E}\left[Z_{i}\right]=\sum_{\alpha \in\{0,1\}^{n}}|\hat{g}(\alpha)| \operatorname{sign}(\hat{g}(\alpha)) \chi_{\alpha}(x) /\|\hat{g}\|_{1}=g(x) /\|\hat{g}\|_{1} .
$$

So by Lemma 4, we have

$$
\operatorname{Pr}[\exists x,|h(x)-g(x)|>\delta] \leq 2^{n} \operatorname{Pr}\left[\left|\sum_{i} Z_{i}-\frac{g(x) M}{\|\hat{g}\|_{1}}\right|>\frac{\delta M}{\|\hat{g}\|_{1}}\right] \leq 2^{n+1} e^{-\frac{\delta^{2} M}{4\|\hat{g}\|_{1}^{2}}} \leq \lambda
$$

\subsection{Protocol}

Now we describe the protocol in this section. The setup is as follows. Suppose that there is a function $f:\{0,1\}^{n} \rightarrow\{+1,-1\}$, which can be approximated by a Fourier sparse function $g:\{0,1\}^{n} \rightarrow \mathbb{R}$ satisfying that $\|f-g\|_{\infty} \leq \epsilon$. The Fourier expansion of $g$ is $g=\sum_{\alpha} \hat{g}(\alpha) \chi_{\alpha}$ and let $A=\operatorname{supp}(\hat{g})$. In addition, let $d=\operatorname{deg}_{2}(f)$ and $N=2^{n}$. For each $k \in\{0,1, \ldots, d-1\}$, Alice and Bob fix an encoding $E_{k}:\{0,1\}^{n} \rightarrow\left[|A|^{2^{k}}\right]$ s.t. for any $\alpha, \beta \in 2^{k} A, E_{k}(\alpha) \neq E_{k}(\beta)$. Finally, for a real function $h:\{0,1\}^{n} \rightarrow \mathbb{R}$ define $\Delta_{t} h(x)=h(x) h(x+t)$. The algorithm is in Box Algorithm 1 . 


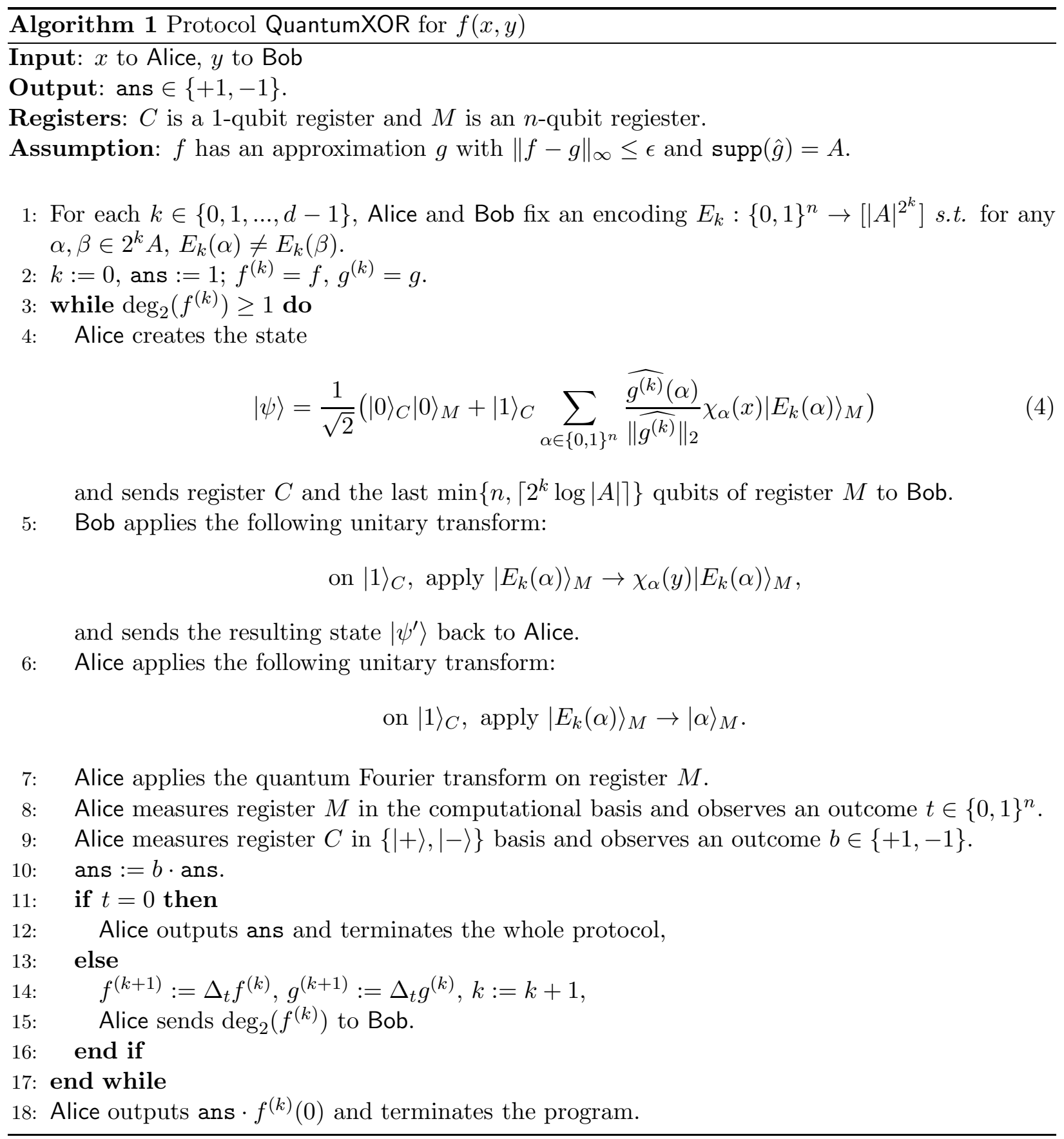


Lemma 6. For any function $f:\{0,1\}^{n} \rightarrow\{+1,-1\}$ and $g:\{0,1\}^{n} \rightarrow \mathbb{R}$, if $\|f-g\|_{\infty} \leq \epsilon$, then for any $t_{1}, \ldots, t_{k} \in\{0,1\}^{n},\left\|\Delta_{t_{1}} \cdots \Delta_{t_{k}} f-\Delta_{t_{1}} \cdots \Delta_{t_{k}} g\right\|_{\infty} \leq(1+\epsilon)^{2^{k}}-1$.

Proof. When taking derivative once, the approximation error increases as follows.

$$
\left|\Delta_{t} f(x)-\Delta_{t} g(x)\right|=|f(x) f(x+t)-g(x) g(x+t)| \leq(1+\epsilon)^{2}-1=2 \epsilon+\epsilon^{2} .
$$

Using an induction, we can easily see that taking $k$ derivatives has the following effect on the accuracy.

$$
\left\|\Delta_{t_{1}} \cdots \Delta_{t_{k}} f-\Delta_{t_{1}} \cdots \Delta_{t_{k}} g\right\|_{\infty}=(1+\epsilon)^{2^{k}}-1
$$

Lemma 7. Suppose that $f:\{0,1\}^{n} \rightarrow\{+1,-1\}$ and $g:\{0,1\}^{n} \rightarrow \mathbb{R}$ has $\|f-g\|_{\infty} \leq \epsilon<2^{-d-1}$, where $d=\operatorname{deg}_{2}(f)$. Then Protocol QuantumXOR computes $f(x+y)$ by at most $2^{d+2} \log \|\hat{g}\|_{0}$ qubits of communication, and the error probability is at most $2^{d} \epsilon$.

Proof. Let us analyze the protocol step by step. (For the convenience of understanding, first think of $k=0$ in the following.) In Step (4), it is easy to see that the $\ell_{2}$-norm of the state is $\frac{1}{2}+\frac{1}{2} \sum_{\alpha}\left|\widehat{g^{(k)}}(\alpha)\right|^{2} /\left\|\widehat{g^{(k)}}\right\|_{2}^{2}=1$, thus the state in Eq.(4) is indeed a quantum pure state. After Step (5), the state is

$$
\left|\psi^{\prime}\right\rangle=\frac{1}{\sqrt{2}}\left(|0\rangle_{C}|0\rangle_{M}+|1\rangle_{C} \sum_{\alpha \in\{0,1\}^{n}} \frac{\widehat{g^{(k)}}(\alpha)}{\left\|\widehat{g^{(k)}}\right\|_{2}} \chi_{\alpha}(x+y)\left|E_{k}(\alpha)\right\rangle_{M}\right) .
$$

After decoding $\alpha$ in Step (66) and applying the quantum Fourier transform in Step (7), Alice holds the state

$$
\begin{aligned}
\left|\psi^{\prime \prime}\right\rangle & =\frac{1}{\sqrt{2 N}}\left(|0\rangle_{C} \sum_{t \in\{0,1\}^{n}}|t\rangle_{M}+|1\rangle_{C} \sum_{\alpha \in\{0,1\}^{n}, t \in\{0,1\}^{n}} \frac{\widehat{g^{(k)}}(\alpha)}{\widehat{\left\|g^{(k)}\right\|_{2}}} \chi_{\alpha}(x+y) \chi_{\alpha}(t)|t\rangle_{M}\right) \\
& =\frac{1}{\sqrt{N}} \sum_{t \in\{0,1\}^{n}} \frac{1}{\sqrt{2}}\left(|0\rangle_{C}+\sum_{\alpha \in\{0,1\}^{n}} \frac{\widehat{g^{(k)}}(\alpha)}{\widehat{\| g^{(k)}} \|_{2}} \chi_{\alpha}(x+y+t)|1\rangle_{C}\right)|t\rangle_{M} \\
& =\frac{1}{\sqrt{N}} \sum_{t \in\{0,1\}^{n}} \frac{1}{\sqrt{2}}\left(|0\rangle_{C}+\frac{g^{(k)}(x+y+t)}{\left\|\widehat{g^{(k)}}\right\|_{2}}|1\rangle_{C}\right)|t\rangle_{M} .
\end{aligned}
$$

After the measurement in Step (8), Alice obtains a random direction $t$, and the state left in register $C$ is $\frac{1}{\sqrt{2}}\left(|0\rangle_{C}+\frac{g^{(k)}(x+y+t)}{\left\|g^{(k)}\right\|_{2}}|1\rangle_{C}\right)$. Then in the next step, measuring register $C$ in the $\{+1,-1\}$ basis gives $f^{(k)}(x+y+t)$ with high probability. Indeed, by Parseval's Identity,

$$
\left\|\widehat{g^{(k)}}\right\|_{2}=\left\|g^{(k)}\right\|_{2}=\sqrt{\mathbf{E}_{x}\left[g^{(k)}(x)^{2}\right]} \leq(1+\epsilon)^{2^{k}} .
$$

Thus when Alice measures $C$, she observes $\frac{1}{\sqrt{2}}\left(|0\rangle+f^{(k)}(x+y+t)|1\rangle\right)$ with probability

$$
\left(\frac{1}{2}+\frac{f^{(k)}(x+y+t) g^{(k)}(x+y+t)}{2\left\|\widehat{g^{(k)}}\right\|_{2}}\right)^{2} \geq\left(\frac{1}{2}+\frac{1-\left((1+\epsilon)^{2^{k}}-1\right)}{2(1+\epsilon)^{2^{k}}}\right)^{2}=(1+\epsilon)^{-2^{k}} \geq 1-2^{k} \epsilon
$$


where the first inequality uses Lemma 6 and Eq.(51).

Now we explain Step (10) and (12). If $t$ happens to be 0 , then Alice already gets $g^{(k)}(x+y)$ which well approximates $f^{(k)}(x+y)$. In general $t \neq 0$. Turning around the definition of derivative, $\Delta_{t} f(x+y)=f(x+y) f(x+y+t)$, we have that $f(x+y)=f(x+y+t) \Delta_{t} f(x+y)$. Since we have obtained $f(x+y+t)$, the problem of computing $f(x+y)$ reduces to that of computing $\Delta_{t} f$ on the same input $x+y$. This reduction is implemented in Step (10), and we let $f^{(k+1)}=\Delta_{t} f^{(k)}$ and go to the next iteration. Therefore, each round reduces the problem to computing the derivative, which is a lower degree polynomial, on the same input. Finally, when the degree of the polynomial is 0 , the function is constant, thus Alice can easily compute it as the last line after the while loop in the algorithm.

One issue in this approach is that only Alice knows $t$ after Step 8 , but Bob does not know $t$ and consequently does not know $f^{(k+1)}=\Delta_{t} f^{(k)}$ for the next round. Also note that it is unaffordable for Alice to send the whole $t$ to Bob. Therefore, it seems hard for Alice and Bob to coordinate on $E_{k}$. The solution here is to note that for all $h:\{0,1\}^{n} \rightarrow \mathbb{R}$, and for all $t \in\{0,1\}^{n}$, we have

$$
\operatorname{supp}\left(\widehat{\Delta_{t} h}\right) \subseteq \operatorname{supp}(\hat{h})+\operatorname{supp}(\hat{h}) .
$$

Indeed, denote $h_{t}(x)=h(x+t)$, then

$$
\widehat{h_{t}}(\alpha)=\mathbf{E}_{x}\left[h(x+t) \chi_{\alpha}(x)\right]=\mathbf{E}_{x}\left[h(x) \chi_{\alpha}(x) \chi_{\alpha}(t)\right]=\chi_{\alpha}(t) \hat{h}(\alpha) .
$$

Therefore,

$$
\widehat{\Delta_{t} h}(\alpha)=\widehat{h \cdot h_{t}}(\alpha)=\sum_{\beta} \hat{h}(\beta+\alpha) \hat{h}_{t}(\beta)=\sum_{\beta} \hat{h}(\beta+\alpha) \hat{h}(\beta) \chi_{t}(\beta) .
$$

If $\alpha \notin \operatorname{supp}(\hat{h})+\operatorname{supp}(\hat{h})$, then there is simply no $\beta$ s.t. both $\hat{h}(\beta)$ and $\hat{h}(\beta+\alpha)$ are nonzero. This implies that $\operatorname{supp}\left(\widehat{\Delta_{t} h}\right) \subseteq \operatorname{supp}(\hat{h})+\operatorname{supp}(\hat{h})$. Using the same argument, it is easily seen that in general, for any $t_{1}, \ldots, t_{k} \in\{0,1\}^{n}$, the derivative $g^{(k)}=\Delta_{t_{1}} \cdots \Delta_{t_{k}} g$ has Fourier support contained in $2^{k} A$. Observe that the only operation Bob makes in each round $k$ is to add a phase $\chi_{\alpha}(y)$ on $\left|E_{k}(\alpha)\right\rangle$. So Alice and Bob can fix an encoding $E_{k}:\{0,1\}^{n} \rightarrow\left[|A|^{2^{k}}\right]$ s.t. $E_{k}(\alpha) \neq E_{k}(\beta)$ for any $\alpha, \beta \in 2^{k} A$. 1 Since for any $t_{1}, \ldots, t_{k} \in\{0,1\}^{n}, E_{k}\left(\operatorname{supp}\left(g^{(k)}\right)\right) \subseteq\left[|A|^{2^{k}}\right]$, the encoding $E_{k}$ is injective on $\operatorname{supp}\left(g^{(k)}\right)$, and thus Alice and Bob can decode in Steps (5) and (6). This also explains why Alice only needs to send the last $\min \left\{n,\left\lceil 2^{k} \log |A|\right\rceil\right\}$ qubits of register $M$ to Bob in Step (44).

Next we analyze the error probability. The protocol is correct as long as in each iteration $k$, the observed outcome $b$ in Step (9) is equal to $f^{(k)}(x+y)$. Since each iteration $k$ has error probability $2^{k} \epsilon$ as showed in Eq.(6), applying the union bound over $k=1,2, \ldots, d-1$ gives that the probability that there exists one round $k$ in which the output bit disagrees with $\Delta_{t_{1}, \ldots, t_{k-1}} f\left(x+y+t_{k}\right)$ is at $\operatorname{most} \sum_{k=0}^{d-1} 2^{k} \epsilon \leq 2^{d} \epsilon$.

Finally we analyze the communication cost. In the while loop, only Step (4) and (5) need communication of $1+\left\lceil 2^{k} \log |A|\right\rceil$ qubits each. Since taking derivative decreases the $\mathbb{F}_{2}$-degree by at least 1 , we know that $k \leq \operatorname{deg}_{2}(f)-1$ before the while loop ends. The total communication cost is at most

$$
\sum_{k=0}^{d-1} 2\left(1+\left\lceil 2^{k} \log |A|\right\rceil\right) \leq 2^{d+1} \log |A|+2 d<2^{d+2} \log |A|
$$

\footnotetext{
${ }^{1}$ It is admittedly true that for a particular set of directions $t_{1}, \ldots, t_{k} \in\{0,1\}^{n}$, the Fourier spectrum for $g^{(k)}=$ $\Delta_{t_{1}} \cdots \Delta_{t_{k}} g$ is only a subset of $2^{k} A$, thus $\hat{g}^{(k)}(\alpha)=0$ for some $\alpha \in 2^{k} A$. But this does not affect the correctness of the protocol, though some communication is wasted in coping with Bob's ignorance of $t$.
} 
qubits.

Now we are ready to prove Theorem 1 .

Theorem 1 (Restated). For any function $f:\{0,1\}^{n} \rightarrow\{0,1\}$ with $\operatorname{deg}_{2}(f)=d$, and any $\epsilon \in$ $\left(0,1 / 2^{d+4}\right)$, we have

$$
\Omega\left(\log \|\hat{f}\|_{1, \epsilon}\right) \leq \mathrm{Q}_{\epsilon}(f \circ \oplus) \leq O\left(2^{d}\left(\log \|\hat{f}\|_{1, \epsilon}+\log \frac{n}{\epsilon}\right) \log (1 / \epsilon)\right) .
$$

Proof. The lower bound is from [LS09]. For the upper bound, by definition, there is a function $g:\{0,1\}^{n} \rightarrow \mathbb{R}$ with $\|f-g\|_{\infty} \leq \epsilon$ and $\|\hat{g}\|_{1}=\|\hat{f}\|_{1, \epsilon}$. We first use Lemma 5 to get a function $h$ with $\|f-h\|_{\infty} \leq 2 \epsilon$ and $\|\hat{h}\|_{0} \leq O\left(\|\hat{g}\|_{1}^{2} n / \epsilon^{2}\right)$. Then we use the protocol QuantumXOR and Lemma 7 to obtain a protocol of error probability $2^{d+2} \epsilon \leq 1 / 4$ and communication cost $O\left(2^{d} \log \|\hat{h}\|_{0}\right)=O\left(2^{d}\left(\log \|\hat{g}\|_{1}+\log \frac{n}{\epsilon}\right)\right)$. Repeat the protocol for $\bar{k}=O(\log (1 / \epsilon))$ times to reduce the error probability to $\epsilon$, and the communication cost is

$$
O\left(2^{d}\left(\log \|\hat{g}\|_{1}+\log \frac{n}{\epsilon}\right) \log (1 / \epsilon)\right)=O\left(2^{d}\left(\log \|\hat{f}\|_{1, \epsilon}+\log \frac{n}{\epsilon}\right) \log (1 / \epsilon)\right) .
$$

Given the above proof, Theorem 3 is an easy corollary.

Theorem 3 (Restated). For any function $f:\{0,1\}^{n} \rightarrow\{0,1\}$ with $\operatorname{deg}_{2}(f)=d$, we have

$$
\frac{1}{2} \log _{2}\|\hat{f}\|_{0} \leq \mathrm{Q}_{E}(f \circ \oplus) \leq 2^{d+1} \log _{2}\|\hat{f}\|_{0} .
$$

In particular, $\mathrm{Q}_{E}(f \circ \oplus)$ is polynomially related to $\log \operatorname{rank}\left(M_{f \circ \oplus}\right)$ when $\operatorname{deg}_{2}(f)=O\left(\log \log \|\hat{f}\|_{0}\right)$.

Proof. The lower bound is from [BdW01]. The upper bound is from Lemma 7 by letting $g=f$.

Finally we notice that all the steps, except for the encoding and decoding of $E_{k}$, can be implemented efficiently.

Proposition 8. The protocol in Theorem 3 needs only $O(d n)$ Hadamard gates, $C$-NOT gates and single-qubit measurements, $2^{d}$ calls of $f$, plus the computation for encoding and decoding of $\left\{E_{k}\right\}$.

Proof. The quantum Fourier transform on $\{0,1\}^{n}$ can be implemented by $n$ Hadamard gates, and all other steps except for the encoding and decoding can also be implemented using $O(n)$ CNOT gates and single-qubit measurements. The only step that may need explanation is when Alice prepares the initial state

$$
|\psi\rangle=\frac{1}{\sqrt{2}}\left(|0\rangle_{C}|0\rangle_{M}+|1\rangle_{C} \sum_{\alpha \in\{0,1\}^{n}} \widehat{f^{(k)}}(\alpha) \chi_{\alpha}(x)\left|E_{k}(\alpha)\right\rangle_{M}\right)
$$

This can indeed by implemented easily as follows. Alice prepares $|+\rangle_{C}|0\rangle_{M}$, and conditioned on $C$ being $|1\rangle$, applies quantum Fourier transform on $M$ to get $\frac{1}{\sqrt{N}} \sum_{z}|z\rangle_{M}$. Now Alice adds the phase $f^{(k)}(z)$ on $|z\rangle$ by $2^{k}$ calls to $f$. After applying the quantum Fourier transform again on $M$, Alice obtains the state $\frac{1}{N} \sum_{\alpha} \sum_{z} f^{(k)}(z) \chi_{\alpha}(z)|\alpha\rangle_{M}=\sum_{\alpha} \widehat{f^{(k)}}(\alpha)|\alpha\rangle_{M}$. Then Alice adds the phase $\chi_{\alpha}(x)$ on $|\alpha\rangle_{M}$ and gets $\sum_{\alpha} \widehat{f^{(k)}}(\alpha) \chi_{\alpha}(x)|\alpha\rangle_{M}$. Finally Alice encodes $\alpha$ and gets the state $\sum_{\alpha} \widehat{f^{(k)}}(\alpha) \chi_{\alpha}(x)\left|E_{k}(\alpha)\right\rangle_{M}$, as desired. 


\section{Acknowledgments}

The author would like to thank Ronald de Wolf and Zhaohui Wei for valuable comments on earlier version of the paper. Part of the research was conducted when the author visited Tsinghua University in China (China Basic Research Grant 2011CBA00300, sub-project 2011CBA00301) and Centre of Quantum Technologies in Singapore partially under their support. The research was also supported by Research Grants Council of the Hong Kong S.A.R. (Project no. CUHK418710, CUHK419011).

\section{References}

[Amb96] Andris Ambainis. Communication complexity in a 3-computer model. Algorithmica, 16(3):298-301, 1996.

[BCWdW01] Harry Buhrman, Richard Cleve, John Watrous, and Ronald de Wolf. Quantum fingerprinting. Physical Review Letters, 87(16), 2001.

[BdW01] Harry Buhrman and Ronald de Wolf. Communication complexity lower bounds by polynomials. In Proceedings of the 16th Annual IEEE Conference on Computational Complexity, pages 120-130, 2001.

[BK97] László Babai and Peter G. Kimmel. Randomized simultaneous messages: Solution of a problem of Yao in communication complexity. In IEEE Conference on Computational Complexity, pages 239-246, 1997.

[BS91] J. Bruck and Roman Smolensky. Polynomial threshold functions, $\mathrm{AC}^{0}$ functions and spectral norms. In Proceedings of the 32nd Annual IEEE Symposium Foundations of Computer Science, pages 632-641, 1991.

[CR12] Amit Chakrabarti and Oded Regev. An optimal lower bound on the communication complexity of Gap-Hamming-Distance. SIAM Journal on Computing, 41(5):12991317, 2012.

[DP12] Devdatt Dubhash and Alessandro Panconesi. Concentration of Measure for the Analysis of Randomized Algorithms. Cambridge University Press, 2012.

[GKdW04] Dmitry Gavinsky, Julia Kempe, and Ronald de Wolf. Quantum communication cannot simulate a public coin. arXiv:quant-ph/0411051, 2004.

[Gro97] Vince Grolmusz. On the power of circuits with gates of low L1 norms. Theoretical Computer Science, 188(1-2):117-128, 1997.

[HSZZ06] Wei Huang, Yaoyun Shi, Shengyu Zhang, and Yufan Zhu. The communication complexity of the Hamming Distance problem. Information Processing Letters, 99(4):149$153,2006$.

[JKS08] T. S. Jayram, Ravi Kumar, and D. Sivakumar. The one-way communication complexity of Hamming Distance. Theory of Computing, 4(6):129-135, 2008. 
[KS13] Raghav Kulkarni and Miklos Santha. Query complexity of matroids. In Proceedings of the 8th International Conference on Algorithms and Complexity, 2013.

[LLZ11] Ming Lam Leung, Yang Li, and Shengyu Zhang. Tight bounds on the communication complexity of symmetric XOR functions in one-way and SMP models. In Proceedings of the 8th Annual Conference on Theory and Applications of Models of Computation, pages 403-408, 2011.

[LS88] László Lovász and Michael E. Saks. Lattices, Möbius functions and communication complexity. In Proceedings of the 29th Annual Symposium on Foundations of Computer Science, pages 81-90, 1988.

[LS09] Troy Lee and Adi Shraibman. Lower bounds on communication complexity. Foundations and Trends in Theoretical Computer Science, 3(4):263-398, 2009.

[LZ10] Troy Lee and Shengyu Zhang. Composition theorems in communication complexity. In Proceedings of the 37th International Colloquium on Automata, Languages and Programming (ICALP), pages 475-489, 2010.

[LZ13] Yang Liu and Shengyu Zhang. Quantum and randomized communication complexity of XOR functions in the SMP model. ECCC, 20(10), 2013.

[MO10] Ashley Montanaro and Tobias Osborne. On the communication complexity of XOR functions. arXiv:, 0909.3392v2, 2010.

[MS82] Kurt Mehlhorn and Erik M. Schmidt. Las Vegas is better than determinism in VLSI and distributed computing (extended abstract). In Proceedings of the fourteenth annual ACM symposium on Theory of computing, pages 330-337, 1982.

[NS96] Ilan Newman and Mario Szegedy. Public vs. private coin flips in one round communication games. In Proceedings of the Twenty-Eighth Annual ACM Symposium on the Theory of Computing, pages 561-570, 1996.

[She12] Alexander A. Sherstov. The communication complexity of Gap Hamming Distance. Theory of Computing, 8(8):197-208, 2012.

[SW12] Xiaoming Sun and Chengu Wang. Randomized communication complexity for linear algebra problems over finite fields. In Proceedings of the 29th International Symposium on Theoretical Aspects of Computer Science, pages 477-488, 2012.

[TWXZ13] Hing Yin Tsang, Chung Hoi Wong, Ning Xie, and Shengyu Zhang. Fourier sparsity, spectral norm, and the Log-rank Conjecture. In Proceedings of the 54th Annual IEEE Symposium Foundations of Computer Science, 2013.

[Vid12] Thomas Vidick. A concentration inequality for the overlap of a vector on a large set, with application to the communication complexity of the Gap-Hamming-Distance problem. Chicago Journal of Theoretical Computer Science, 2012(1), July 2012.

[Yao79] Andrew Chi-Chih Yao. Some complexity questions related to distributive computing. In Proceedings of the Eleventh Annual ACM Symposium on Theory of Computing (STOC), pages 209-213, 1979. 
[Yao03] Andrew Chi-Chih Yao. On the power of quantum fingerprinting. In Proceedings of the Thirty-Fifth Annual ACM Symposium on Theory of Computing, pages 77-81, 2003.

[ZS09] Zhiqiang Zhang and Yaoyun Shi. Communication complexities of symmetric XOR functions. Quantum Information 83 Computation, 9(3):255-263, 2009.

[ZS10] Zhiqiang Zhang and Yaoyun Shi. On the parity complexity measures of Boolean functions. Theoretical Computer Science, 411(26-28):2612-2618, 2010. 